\title{
A Model of Clinical Intervention in the Maternity Ward: The Breastfeeding and Bonding EMDR Protocol
}

\author{
Valentina Chiorino \\ Sara Roveraro \\ M. Caterina Cattaneo \\ Roberta Salerno \\ Elena A. Macchi \\ Giorgia G. Bertolucci \\ Perinatal Psychology and Postpartum Unit, Milan, Italy \\ Fabio Mosca \\ Neonatal Intensive Care Unit Department, University of Milan, Italy \\ Isabel Fernandez \\ Psychotraumatology Research Center, Milan, Italy
}

\begin{abstract}
Breastfeeding is one of the main manifestations of the bond that a mother builds with her newborn baby. Literature on psychological support for mothers in the early stages of breastfeeding is limited and interventions often do not pinpoint the actual roots of the difficulties. Breastfeeding difficulties may cause emotional distress to women and this can impact significantly on bonding and the perinatal period may turn into a state of crisis. Therefore, it is essential for the clinical psychologist to intervene selectively and in a prompt, effective way, especially when working in a maternity ward. This article suggests a model of intervention: the Breastfeeding and Bonding EMDR Protocol. This protocol, created ad hoc for breastfeeding, combines the work with eye movement desensitization and reprocessing (EMDR) on recent events, the standard protocol and the installation of resources. The hospital case study presented here thoroughly illustrates the various stages of the protocol and the peculiarity and functionality of EMDR regarding breastfeeding and bonding issues in the immediate postpartum period. Prevention is the paramount subject of the model of clinical intervention on breastfeeding hereafter presented.
\end{abstract}

Keywords: EMDR; recent event; breastfeeding; bonding; resources; birth

W omen can experience a range of psychological problems after birth, including anxiety, depression, and adjustment disorders (Beck, 2006; Coates, Ayers, \& de Visser, 2014; O’Hara \& Swain, 1996). Researchers have shown that in the postpartum period, anxiety symptoms and anxiety disorders are as common as depression (Matthey, Barnett, Ungerer, \& Waters, 2000; Ross \& McLean, 2006). In the immediate postpartum period, breastfeeding difficulties are one of the reasons of emotional distress for women and may impact significantly on the bonding between mother and infant and alter the building of the attachment relationship (Glasheen, Richardson, \& Fabio, 2010; Groer, Davis,
\& Hemphill, 2002; Nicol-Harper, Harvey, \& Stein, 2007).

The purpose of this article is to explain eye movement desensitization and reprocessing (EMDR) clinical intervention, by implementing an ad hoc protocol for breastfeeding in a maternity ward of a hospital to offer a new model to treat problems with breastfeeding besides those reported in literature, which are principally based on generic supportive intervention and self-efficacy promotion (Burns, Schmied, Fenwick, \& Sheehan, 2012; Dyson, McCormick, \& Renfrew, 2006; Kingston, Dennis, \& Sword, 2007; Renfrew, McCormick, Wade, Quinn, \& Dowswell, 2012; Schmied, Beake, Sheehan, McCourt, \& Dykes, 2011). 


\section{Introduction: The Bonding Phenomenology}

"The 12A has been crying since yesterday, she can't hold her baby, she can't breastfeed her . . . she feels like fainting as soon as the baby latches on ... can you go and see her?" This is how a psychological intervention on breastfeeding starts in a maternity ward, after the warning of a midwife who spots an unexpected, acute, and apparently unjustified anguish toward taking care of the newborn, some sort of "panic attack" related to taking care. Facing a new role that involves unfamiliar demands, including breastfeeding, the new mother can develop several kinds of anxiety disorders (performance anxiety, anxiety about her child's health, anxiety about her body and its changes ... ), which can sometimes turn into high level of distress and uneasiness. Today, most mothers discontinue breastfeeding before the first 6 months, which is the minimum period recommended by the American Academy of Pediatrics (2014) and far before the even longer duration suggested by the World Health Organization (2016). A major reason of the early discontinuation is difficulty with breastfeeding rather than maternal choice (Barber, Abernathy, Steinmetz, \& Charlebois, 1997; Hill, 1991).

\section{The Early Stages of Breastfeeding for New Mothers: Which Kinds of Difficulties?}

The process of breastfeeding takes place as bonding happens. Bonding is a physical, emotional, and cognitive connection, which develops between the mother and her baby (Else-Quest, Hyde, \& Clark, 2003). Bonding starts during pregnancy and grows and strengthens after the birth of the baby (Siddiqui, Hagglof, \& Eisemann, 2000); in this perspective, breastfeeding can be perceived as one of the main expressions of bonding. It is not simply a question of feeding, but indeed of creating that bond between mother and child (Winnicott, 1987). Breastfeeding mothers tend to be calmer, less anxious, and less stressed than formulafeeding mothers; to be more responsive to their infants; to touch them more during both feedings and playtime (Jansen, de Weerth, \& Riksen-Walraven, 2008; Kuzela, Stifter, \& Worobey, 1990; Mezzacappa, Guethlein, Vaz, \& Bagiella, 2000; Tharner et al., 2012; Wiesenfeld, Malatesta, Whitman, Granrose, \& Uili, 1985). Furthermore, breastfeeding dyads spend significantly more time in mutual gaze during feedings than do bottlefeeding dyads (Lavelli \& Poli, 1998). Researches show that early breastfeeding is associated with less parental negative affect, less parental intrusiveness, and less infant dysregulation at 12 and 14 months (Else-Quest et al., 2003; Tharner et al., 2012). These findings indicate that breastfeeding may be associated with the maternal sensitive responsiveness and the quality of the infant-mother bonding process. There is now considerable evidence, from human and animal studies, that oxytocin and prolactin, hormones that are released during breastfeeding mainly to support milk production and secretion, facilitate maternal caregiving behavior and increase parental sensitive responsiveness toward the child (Francis, Champagne, \& Meaney, 2000; Mann \& Bridges, 2001). Recent studies in humans have shown that oxytocin is related to a reduced levels of stress, to an increased level of empathy (Kim et al., 2011; Rodrigues, Saslow, Garcia, John, \& Keltner, 2009), and to a higher pronounced physiological reactivity (heart rate) to infant crying (Riem, Pieper, Out, Bakermans-Kranenburg, \& van IJzendoorn, 2011).

Non-nutritive aspects of breastfeeding, such as close bodily contact and direct feedback via nipple stimulation, may also be associated with sensitive responsiveness, for example, by enhancing attunement of emotional and attentive states between infant and mother. Maternal care is a complex set of behaviors and attitudes. Therefore, it can be inferred that a psychological intervention on breastfeeding can positively affect attunement and bonding.

Generally, the initial experience of breastfeeding starts when a mother is in hospital, either a few moments after delivery or within hours, sometimes even 1 day after her child's birth (Adewale, 2006). The very first skin-to-skin contact between mother and infant and the first time the baby grasps his or her mother's nipple are crucial for a mother. What does a mother feel when her newborn promptly grasps her nipple, or when he or she dozes, not seeming interested in nursing? And what happens, on the contrary, when the child is restless, when he or she cries and struggles to latch on, failing, or when it seems that he or she does not like it (Hegney, Fallon, \& O’Brien, 2008; Williamson, Leeming, Lyttle, \& Johnson, 2012)? The crucial point is that all these unforeseen situations or initial difficulties in breastfeeding and taking care of the newborn, which previously had not been taken into account, are felt as actual failures. These difficulties bring with them feelings of inadequacy, incompetence, self-defectiveness, and powerlessness, despite the fact that they are absolutely common (Hailes $\&$ Wellard, 2000; Hauck, Hall, \& Jones, 2007; Hauck, Langton, \& Coyle, 2002; Li, Fein, Chen, \& GrummerStrawn, 2008; Missonnier, 2003).

In the early stages, the mother may feel pain during breastfeeding as the baby latches on vigorously or poorly (without having the entire areola in his or her mouth). In certain cases, the way the newborn sucks 
can lead to the formation of rhagades (Colin $\&$ Scott, 2002). The onset of lactation changes the breasts suddenly and enormously and can generate a sense of lack of control on the body (Brodribb, 2012). Sometimes, women think that their body is not working properly or is unsuitable for breastfeeding (Ballard, Stanley, \& Brockington, 1995; Cousineau et al., 2006). It must be said that in some cases, the shape of the nipple, for example, when it is inverted, can make it still more complicated for a mother to breastfeed (Colin \& Scott, 2002). The "physiological weight loss in the neonate" (as defined by pediatricians) generates a sense of danger and fear for the poor state of health of the child, and even helplessness because there is still no milk (Cattaneo, Roveraro, Chiorino, Salerno, et al., 2015). In some women, the idea of danger appears even when a baby regurgitates some milk because they fear he or she could suffocate.

A sense of inadequacy is felt when, for some reason, a mother feels unable to deal with the new task in the way she wanted or had expected. The experience of inadequacy may also affect the process of taking care in general because a mother may think she cannot console her baby when he or she is crying, calm him or her down when he or she is upset, or understand his or her needs. These initial difficulties, which are natural and legitimate elements of the process of familiarization, are often seen as insurmountable obstacles when combined with a feeling of inevitable failure. A state of crisis may develop caused by the personal feeling of being judged "a bad mother" because of the inability to nurse. Those feelings often become the first signs of a risk of depression (postpartum depression; Cattaneo, Roveraro, Chiorino, Macchi, et al., 2015; Groer et al., 2002).

\section{From the Initial Difficulties Concerning Breastfeeding to Negative Cognitions}

Because mothers breastfeed several times throughout the day, in numerous cases, the difficulties described earlier, which emerge in the first hours and in the early days of taking care of the newborn, often happen again and can worsen, too. Mothers can experience a state of growing anxiety, sometimes despair, or emotional confusion (Hegney et al., 2008). At this time, several dysfunctional thoughts may begin to take shape, causing a negative impact on breastfeeding, on the psychophysical health of the woman, on taking care of the newborn, on bonding, and on the beginning of attachment process in general. Mothers can begin to think poorly of themselves: "I am inadequate," "I am not going well," "I am powerless," "I lack control."
The sense of danger tends to be felt in relation to the child's weight loss. In this article, the authors will highlight some negative cognitions (NCs) that they have identified as specific to breastfeeding, besides the classic ones (Cattaneo, Roveraro, Chiorino, Salerno, et al., 2015). Primary prevention consists of measures of intervention, which precede the crystallization of negative thoughts about the mother herself and/or her infant and/or her relationship with him or her.

\section{The Clinical Psychologist Within the Hospital Context and the Hospital Setting}

In hospital, the crisis of a mother is usually pointed out by a midwife, a pediatrician, a baby nurse, or a gynecologist (and sometimes by the mother herself). However, an intervention by a clinical psychologist to address a breastfeeding problem is usually requested from the second day, and rarely before. Considering the short time available before the discharge takes place, it is clear that the intervention must be focused and brief. In most cases, it will be possible to conduct one or maximum two sessions with the woman directly in her room, while she tends to lie on her bed with the baby (asleep or calm or even desperately crying) next to her or in the cot, involving her partner and maybe in front of the roommate, while the rhythm of the ward goes on with its medical examinations, visits, blood tests, and so on. Because the woman did not ask for a psychological assessment, that on the contrary was suggested by the hospital staff or proposed to her when a nursing issue occurred, the intervention is conducted even in the absence of an initial psychological demand, and therefore, the therapeutic alliance deserves particular attention. If breastfeeding becomes the main issue and the subject of the intervention, it will be easier for the woman, who is aware of her difficulties with the new task, to better understand the aim of the session and to establish a rapid therapeutic alliance based on a clear purpose.

Timing has a strong effect. The urgency of a mother to assist her baby crying, the pressure exercised by her relatives and the hospital staff, and the idea that the onset of lactation should start immediately may lead the woman to lose self-confidence and trust in her own resources.

\section{Breastfeeding and Bonding in Clinical Practice: The Importance of Identifying Potentially Traumatic and Distressing Issues}

At the start of a crisis, as soon as women's difficulties in breastfeeding begin, even one long psychological session can be decisive to reduce the potential of 
risk within it. In the maternity ward, the psychologist's task should be an early assessment: highlighting the critical knots about breastfeeding and offering the chance to reprocess them and to treat the problems.

Critical knots (as defined by the authors of this article) are psychological difficulties and problems that women experience when dealing with breastfeeding. They can be found when the patient's life meets the specific experience of breastfeeding and taking care of the newborn. Some elements of the patient's life can emerge and interfere with the process of breastfeeding: NCs about the self, already structured during one's life, emotions or physical sensations already experienced in the past, can be specifically activated and recalled by the breastfeeding situation, according to tenets of the adaptive information processing (AIP) model (F. Shapiro, 2001). To say it with that model, cognitions, emotions, and sensations, rising during difficult breastfeeding, can trigger older memories, which were processed in a maladaptive way, and create inappropriate thoughts and behavior as well as overt symptoms (high anxiety or depressed mood).

Some examples are given to explain the concept; a mother feels a sense of inadequacy because it seems to her that she is unable to breastfeed, place the baby at her breast, calm him or her down, and feed him or her. The memory network of negative experiences related to that sense of inadequacy is stimulated and comes to mind. If a woman has a negative belief about herself concerning inadequacy and coming from her history (her mother made her felt that way in many situations when she was a little girl and in adolescence), it is clear that she will more likely experience a crisis when facing what she thinks to be the confirmation of her inadequacy. Probably, she will be dejected and will lack confidence in herself and her skills to carry out the new role. Perhaps, childbirth has already fueled the belief of inadequacy, for example, when, during the expulsive phase of labor, the woman was not able to push effectively, and therefore, a vacuum extractor was used or a cesarean section was performed.

\section{Eye Movement Desensitization and Reprocessing}

\section{A Clinical Intervention Focused on Breastfeeding: The Link Between the Session and EMDR}

EMDR is a psychotherapy treatment that facilitates the accessing and processing of traumatic memories and other adverse life experience to bring these to an adaptive resolution. During EMDR, the client attends to emotionally disturbing material while simultaneously focusing on an external stimulus: Lateral eye movements are the most commonly used, but there are other bilateral stimulations (BLS) such as hand tapping or audio stimulation. EMDR facilitates the accessing of the traumatic memory network: The information processing is enhanced, and new associations are forged between the traumatic memory and more adaptive memories or information. New learning, elimination of emotional distress, and development of cognitive insights are the main consequences. NCs, which are negative beliefs about self, are reformulated. EMDR is a three-pronged protocol: Past events that have laid the ground for dysfunction are processed, the current circumstances that elicit the distress are targeted, and imaginal templates of future events are incorporated (F. Shapiro, 2001).

The authors choose to introduce EMDR therapy (F. Shapiro, 2001; Solomon \& Shapiro, 2008) into the sessions which take place in the maternity ward to treat breastfeeding and bonding issues (Madrid, Skolek, \& Shapiro, 2006) because EMDR is a powerful and focused intervention at a time when the therapist cannot afford to wait for the effects of a long-term one. EMDR can be even more effective during the immediate postpartum because, at this time, mothers' emotional knots, which had not been treated earlier, emerge in a much clearer and more defined way than during other stages of life and can generate dysfunctional behaviors. EMDR satisfies another essential need. It helps not to label as dysfunctional something which can be normal, allowing healthy transformative processes, reactivating the mind's natural reparative capability, and reconnecting the patient with his or her own resources and potentiality, designed to face this specific time of life. At the same time, EMDR therapy allows the psychologist to pinpoint and work on the issues, which could become a dysfunction. Moreover, EMDR carefully takes into account the patient's body, more than many other therapeutic approaches. This is fundamental because bonding and breastfeeding are experiences strictly connected to the body, its transformations, and the skin-to-skin contact between mother and baby. EMDR uses the location of body sensations as one of the dimensions of the assessment phase and verifies this dimension during the process with EMDR. During an EMDR session, a mother can feel quieter and less anxious; her body relaxes. She can notice the direct effect that the intervention has on the baby, who calms down when handled with less agitation and more firmness. The mother finds a bit more confidence in herself during the EMDR phases. 
The novelty of the Breastfeeding and Bonding EMDR Protocol is the implementation of EMDR approach to the difficulties of breastfeeding as soon as they begin to appear, in the context of a hospital ward. Early EMDR Intervention (EEI) proposes protocols, such as the Recent-Traumatic Episode Protocol (R-TEP; E. Shapiro, 2009a; E. Shapiro \& Laub, 2009), that work on recent traumatic events (that have been experienced few hours or few days before). R-TEP is a comprehensive current trauma-focused protocol for EEI that incorporates and extends the existing eye movement desensitization (EMD), EMDR, and recent event protocols together with additional measures for containment and safety. In R-TEP, the original traumatic incident with its aftermath is viewed as an ongoing trauma continuum, whereas the experiences are not yet adaptively reprocessed. The T-episode comprises multiple targets of disturbance.

The Breastfeeding and Bonding EMDR Protocol addresses the difficulty and the state of crisis connected to breastfeeding similarly to a distressful recent event. The authors refer not only to breastfeeding but also to bonding because, when the intervention is on breastfeeding, the effects can be on attunement and bonding, too, because they are interconnected, as literature shows. Moreover, the Protocol is proposed to women who are in a state of crisis because of their difficulties with breastfeeding, and it is not used when women have difficulties with the new task but they are calm and they do not show any particular psychological reaction (no anxiety, no weeping, no sadness, no anger . . . ). Of course, several women who do not breastfeed or who have some difficulties with breastfeeding are serene and bonding goes smoothly. The risk factor for bonding is the emotional burden that may (but also may not) appear when a woman has breastfeeding difficulties. Because breastfeeding is an ongoing, necessary process, the feeding routine continues fueling the potential current traumatic experience. The mother knows that she will have to face the next feeding soon, with the risk of worsening the sense of anguish, agitation, self-defectiveness, helplessness, or danger that she feels. The Protocol also highlights several possible NCs, which are peculiar to the situation of breastfeeding; these are the result of the clinical practice of the authors of this article. In its final part, the Protocol proposes the Installation of Resources through resource development and installation (RDI) in order for the patient to be emotionally ready to deal with her task, which is a moment of psychological distress that can have a traumatic impact, but is also a challenge and a peak performance.
$\mathrm{RDI}$ is a psychotherapeutic tool to develop and install with EMDR positive resources, useful to cope with a challenging situation. It enhances ego strength. The client identifies positive personal experiences, positive self-acknowledged traits, people in her life with positive qualities or traits, and metaphors or symbols that represent positive qualities. Alternating BLS is used to strengthen, one by one, these positive ego states and enhance their accessibility.

\section{The Session: History Taking, Preparation, and Key Questions About Pregnancy and Delivery}

Hospital requires the psychologist to implement a targeted intervention; therefore, the professional collects a brief history of the person and a brief trauma history. Before introducing EMDR during the psychological session, the authors of this article suggest to ask questions about elements which are particularly important for the case conceptualization during postpartum. (The questions have been developed by the authors during several years of clinical experience in the maternity ward.)

- How is she after delivery? How does she feel? How is it going with her baby?

- How is it going with her breastfeeding? And with taking care of her baby and bonding? What are her feelings with breastfeeding? And with taking care and bonding? What did she think about breastfeeding before pregnancy? And during it? And after? Was she breastfed?

- How was the conception? In other words, it is essential to ask whether the baby was planned, whether he or she arrived after a long time or not, whether it was hard to get pregnant, whether it is the first child, and whether there have been other pregnancies which did not reach full term (because of miscarriages, abortions, or intrauterine fetal deaths). (These questions might also help the woman start talking quite spontaneously about whether she has ever undergone medically assisted procreation $[\mathrm{MAP}]$ and especially artificial insemination by donor.)

- How was pregnancy managed? Were there any worries, anxieties, and physical issues concerning the mother and/or the baby? How did the patient cope with the changes of her body during pregnancy?

- How was delivery? Were there any unexpected traumatic events during delivery?

- How did the couple's relationship evolve during the various stages, from conception to the birth of the baby? 
- Were there any traumatic events in the woman's life? (abuse, unprocessed mourning, ... )

- Has the woman ever suffered from any kind of psychological disorder? Has she ever taken medications for those problems or has she ever gone to a psychotherapist?

These questions can be used as a guideline: They allow the clinical psychologist to set the focus of intervention on the woman's main issue accordingly. This can be particularly useful when implementing a specific intervention with EMDR.

\section{Intervention Method: The Breastfeeding and Bonding EMDR Protocol}

These are the three steps of the protocol:

Step 1: The woman is requested to tell the various moments with her baby out loud, referring to breastfeeding, taking care, and bonding (Narrative), while receiving BLS administered by the therapist. The intention is to lower the level of arousal.

Step 2: EMDR procedures are used to help the woman process the moment (or moments) of greatest difficulty/distress related to breastfeeding, taking care of the newborn and bonding. The intention is to unravel the critical knots that hinder breastfeeding.

Step 3: RDI procedures are used to install and strengthen personal resources. The intention is to help the woman feel more confident and prepared with future breastfeeding.

\section{Application of the Breastfeeding and Bonding EMDR Protocol}

When clinical psychologists meet a woman who has just given birth to a baby and shows difficulties in breastfeeding, they need to verify first whether she has a sufficient ego strength (during history taking). The clinical psychologists also need to determine that any possible trauma found in the anamnesis is not so destabilizing in its present form. Then, they introduce EMDR (preparation phase: a brief explanation of the intervention with EMDR they are proposing) and use the EMDR Protocol for breastfeeding. The Dissociative Experiences Scale (DES; Bernstein \& Putnam, 1986) can be useful to decide whether to proceed with the EMDR Protocol for breastfeeding or not, rapidly identifying dissociative aspects. Moreover, should any dissociative phenomena appear during the treatment with EMDR, or should the woman show even just too powerful a reaction for her to cope with, she will be brought back to a state of control and safety through stabilization techniques such as grounding (e.g., with the exercise of the four elements; E. Shapiro, 2009b) or the safe place (Luber $\&$ Shapiro, 2009). The authors believe the model of clinical intervention described in this article should be performed by psychotherapists with a sound clinical expertise both in general terms and specifically with EMDR and with EEI (E. Shapiro, 2009a; E. Shapiro \& Laub, 2009; F. Shapiro, 2001) and that have sufficient familiarity with treatment in a hospital context and with issues specific to pregnancy and postpartum. However, studies on EEI are limited, and more research must be conducted with controlled studies (Buydens, Wilensky, \& Hensley, 2014; Fernandez, Callerame, Maslovaric, \& Wheeler, 2014; Jarero, Artigas, Uribe, \& Miranda, 2014; Maxfield, 2008).

The treatment of breastfeeding issues with EMDR can focus on past, present, and future situations because the knots of the past emerge powerfully (as in the case study described here) or mostly on present and future situations (but still working on knots which have links to the past). "Future" is the near future; it could be the next time the baby will cry to tell the mother he or she is hungry and/or when the mother will try to latch him or her onto the breast.

\section{First Step With EMDR: The Narrative and Its Function}

The woman is ready to work with the Breastfeeding and Bonding EMDR Protocol, after recollecting her history and trauma history, after being asked specific questions about pregnancy and delivery (see questions in the preceding text; Phase 1), and after being prepared for the EMDR (Phase 2, preparation and stabilizing techniques). The woman, normally lying in a hospital bed with the newborn baby in her arms or lying next to her or asleep in the cot, is requested to tell the various moments with her baby (starting from those she wants) out loud, referring to breastfeeding, taking care, and bonding. While the mother is telling her story, the clinical psychologist performs uninterrupted BLS (E. Shapiro \& Laub, 2009). This is the narration of the beginning of bonding (or its development after the first hints during pregnancy). Some women start the Narrative from the time they found out they were pregnant, some others start from a critical time during pregnancy, and others from delivery and the first time they met their baby, having previously explained that it "increases the sense of safety because of its presumed grounding and de-arousal effects" (E. Shapiro \& Laub, 2009, p. 196). The clinical 
psychologist also clarifies that EMDR is a psychotherapy treatment that facilitates the accessing and processing of traumatic memories and other adverse/ distressing life experience to bring these to an adaptive resolution. He also explains that EMDR improves the flow and the correlation between thoughts, emotions, images, and feelings and facilitates the inclusion of different points of view in the woman's experience (thanks to desensitization and reprocessing). Women notice changes of perspective during the treatment with EMDR. Because the treatment concerns events, which have just occurred, various moments can be revisited, requiring the patient to relive them even more than once. The request is to repeat the Narrative just told, especially if the first one sounded difficult and fragmented, with uninterrupted BLS performed again by the clinical psychologist. The Narrative may be fragmented because the event described by the patient is recent and not structured yet around a main image which would represent it (Guedalia \& Yoeli, 2009). Moreover, problems and difficulties related to breastfeeding and treated with EMDR are not only events that have just happened, but they are also an ongoing process because breastfeeding is obviously continuing.

If the woman feels comfortable with his or her presence, her partner can stay with her even during EMDR intervention as an attentive and sympathetic observer. Besides easing the tension, the Narrative often becomes for the new mother a time in which she can focus again on herself, on the interactions with her baby, and on the bond she is creating with him or her. It is not unlikely that her newborn baby would be quiet or peacefully asleep next to her or that he or she would show signs of hunger and would be looking for the mother's breast. The woman, on the other hand, would be less anxious, calmer. With the use of the BLS (and the next two steps of the protocol), it is thought that the Narrative can favor a feeling of harmony between the mother and her baby and have positive effects on both. It is also possible that the baby could benefit from the higher relaxation that the woman often shows at the end of the session, frequently followed by a feeling of trust in her ability to take care of her child, for whom she is a holder and an emotional platform. As Solomon (2006) describes, EMDR can be seen as a dance between therapist and client, with its speed, rate, and tempo, varying from moment to moment, and it reflects the attunement between them (it is the psychotherapist that calibrates BLS, maximizes the processing, and controls the intensity). This dance during EMDR seems to create at the same time something similar between mother and baby: a sort of promotion of woman's attunement disposal and mood. Witnessing this process step by step can make the partner feel more relaxed as well because he or she can see the woman being somehow more positive and can sense a breakthrough in her feelings of inadequacy and helplessness. The partner will perceive this to a greater extend during the next step.

If it should occur that at the end of the Narrative the mother has not seemed to calm down, as she was initially agitated and distressed, the work of the second step with the processing of the points of greatest difficulty/distress with respect to breastfeeding (see next paragraph) is the best way to handle and untie the critical knots, which are the core of distress and agitation. If the mother does not talk about breastfeeding during her Narrative, the clinical psychologist will tell her to continue with her story, asking her to describe how the first contact with her baby was, how the latch-on started, and how later feedings went.

\section{Second Step: The Critical Knots and the Moments of Greatest Difficulty/Distress Regarding Breastfeeding}

The therapist identifies the critical points, where the thread is twisted and the knots need to be unravelled, asking the woman to scan in her mind the entire story regarding breastfeeding, taking care of her newborn baby, and bonding in no particular order, like a Google Search in the computer, looking for those moments of greatest difficulty/distress. He performs continuous BLS during the Google Search. Then the clinical psychologist asks the new mother, "Which is the worst image that represents that moment?" or "If you think about that moment, what can you observe?" This is followed by the NC (i.e., "I'm powerless"), the positive cognition (PC; "I now have choices"), the 1-7 Validity of Cognition (VoC) scale ("1" equals completely false and " 7 " equals completely true) the Emotions, the 0-10 Subjective Unit of Disturbance (SUD) scale ("0" equals no disturbance; "10" equals the worst feeling you can imagine), and the Location of body sensation (the assessment phase in standard protocols). The psychologist uses as much of the Standard Protocol Assessment Phase as appropriate (NC, PC, VoC, Emotion, SUD, body sensation). Because the patient is dealing with a recent event, "appropriate" could mean that in some cases, the psychologist may choose not to ask for the PC and the VoC during the initial phase and to postpone the identification of the $\mathrm{PC}$ and the request 
for the VoC until the SUD is equal to 0, if he understands that way at that moment is more protective of the hints of therapeutic alliance with the new mother (Parnell, 2007).

Starting from these elements, it is possible to proceed with the EMDR Protocol, through the BLS (the eye movements or tapping), only if, as we already explained earlier, during the course of the Narrative the woman proved to be sufficiently stable emotionally and if there were no apparent great and unprocessed traumas in her history. Then the next points (other moments of difficulty/distress regarding breastfeeding) are to be examined one by one with the protocol.

The authors of this article have pinpointed a few NCs, which are particularly recurring and specific to breastfeeding issues, beside the common NCs that can obviously be used for breastfeeding. A few of these NCs, although suitable for breastfeeding, are similar to the common ones, whereas some others are purely specific to a breastfeeding situation and are founded on the fact that, during the immediate postpartum, the mother feels to be one with her newborn baby. Herewith is the list of some frequent NCs specific for the intervention with EMDR in a perinatal context (Cattaneo, Roveraro, Chiorino, Salerno, et al., 2015).

- My baby is in danger.

- My milk is bad.

- I am not able to protect my child.

- I am a bad mother.

- My breast is defective (or flawed or damaged).

- I cannot breastfeed.

- My baby rejects me/rejects my breast.

- I am not able to understand my child.

- My baby is defective (or flawed or damaged).

- I was not able to create a healthy baby.

- I have no control over my body.

- My body does not function.

The NCs listed earlier can essentially be grouped into the three categories about danger, self-defectiveness, and lack of control/helplessness.

During the phases of desensitization and reprocessing, the authors pay specific attention in taking the new mother back to the initial memory (chosen as representative of the moment of greatest difficulty/ distress), especially if there is the risk for the processing to be haphazard because too many memory channels are stimulated. The therapist takes note of the connections but always bring his or her attention back to the initial memory to keep a tight focus. If the woman is emotionally burdened, the tight focus can help her with the containment of overwhelming feelings. Also when strong physical sensations prevail, the clinical psychologist brings the patient back to the target, in a way much closer to the EMD. However, if applying this method the SUD does not decrease or decreases only partially or if the need to hold the flow of thoughts is not dominant, the therapist will leave more room for the associative process and the opening of memory channels which enable reprocessing and reelaboration (EMDr, EMDR). For this to happen, the therapist does not come back to the target after each set or after a few sets, but she lets the woman go on, even if she moves away from the target and stimulates channels, which can also lead to the connection with other facts concerning breastfeeding (EMDr) or with current facts that do not concern breastfeeding directly, or to elements of the woman's past life (EMDR; E. Shapiro, 2009a; E. Shapiro \& Laub, 2009). Here are some examples. During Step 2, in the desensitization and reprocessing phase of the Breastfeeding and Bonding EMDR Protocol, a woman may begin talking about the sense of inadequacy she feels when trying to breastfeed her child and tell the psychologist (after a set of BLS) that she experiences it when looking at her roommate for whom everything seems to come so easy, or that she felt inadequate when the midwife told her to keep the baby in a different position otherwise he or she cannot latch on properly. Then (after a set of BLS) she may talk about the sense of inadequacy experienced at work recently when dealing with a very judgmental colleague and (after a set of BLS) feel her stomach upset, she may remember the moments (after a set of BLS) in which her mother used to compare her with her sister, who was so good at school, whereas she was not, to finally discuss about (after a set of BLS) her and her mother's satisfaction when she started university and got full marks, graduating on time and with honors. The psychologist will then bring the woman back to the initial target (e.g., at the end of an associative channel) to verify changes. When the psychologist identifies the need to go through associative channels without having to come frequently back to the target because the SUD remains very high and stable, the standard EMDR Protocol is fully implemented. Afterward, the psychologist proceeds with desensitization and reprocessing (Phase 4), installation of PC (Phase 5). The body scan (Phase 6) and the closure (Phase 7) follow the processing of all the moments of greatest difficulty/distress. If the partner witnesses all the stages of the second step, he or she will have a 
chance to better understand the fears and the blocks which his or her partner is experiencing and to develop greater empathy toward her.

\section{Third Step: The Activation of Resources}

Another significant stage is the identification of the resources, which the woman believes could help her deal with breastfeeding and taking care of her child. The new mother can find these resources within herself or draw them from people close to her, who are supporting her, or even from symbols and metaphors. The therapist asks the woman to imagine the next time she will breastfeed her newborn and describe the sequence of images of that moment. Then she invites her to identify which resources, capabilities, and characteristics (her own characteristics or those of someone she knows or of a famous character, or again the characteristics represented by a symbol or a metaphor) she thinks may be useful to face the next feeding. These resources are installed through EMDR, using the RDI (Leeds \& Shapiro, 2000) to make them more accessible for the mother. Some of the most recurring resources a woman identifies as useful for breastfeeding are calm, tranquility, patience, self-confidence, strength, and determination. It is not rare to see these resources represented by supportive people, to whom the woman needs to feel close and with whom she can identify herself. In the specific case of breastfeeding, it is most likely to be a feminine figure, mainly the mother or grandmother or another relative toward whom there is an emotional affinity or a friend who has possibly already experienced motherhood. The therapist will then ask the mother to imagine the next time she will breastfeed her baby and to do it with a PC and using the resources previously identified and installed: This step with EMDR is visualizing the future and is used to link breastfeeding and resources. Her partner can witness the woman recover an idea of resources to carry on with her new role of mother.

The steps of the EMDR Protocol for breastfeeding, especially the second and third one, are actually strongly connected and, in particular situations when the level of discomfort starts to diminish, they can be integrated. This means that the work on resources should be introduced as a part of the work on the moments of greatest difficulty/distress is still ongoing. The more the new mother starts to imagine with confidence the next time she will breastfeed, the more she will be able to integrate the previous stages, which she has experienced as particularly critical, and she will consider them as part of the process of familiarization with breastfeeding and tuning into her baby. The integration between the second and the third step happens when the SUD, although very low, does not reach 0 or any ecological value $(S U D=1)$. The reason expressed by the woman can be that she is worried about the next time she will breastfeed her baby, and she does not know how to face that moment. As explained earlier, beside the moment (or moments) of greater difficulty/distress related to breastfeeding, there is also awareness and fear that other similar events may occur. Working on the future (the next time the woman will breastfeed her newborn) with RDI can be particularly helpful to further process the moment (or moments) of greatest difficulty/distress related to breastfeeding. When the therapist has the chance to meet the woman for a second (or third) session in the ward, they may resume from where they left (from the exact point of the protocol) or, if they already completed the third step (or the second one), the psychologist may work on any other moments of difficulty/distress related to breastfeeding which occurred after the first (or second) session and then come back to-or start from the beginning if they did not address it yet - the work on resources of the third step.

\section{A Case Study}

For the case study presented here, the procedure was as follows. There was a first session in which information about the history of the patient was collected and the Narrative procedure was carried out (first step). In the two further sessions, the second and third steps of the EMDR Protocol, described before, were implemented. It was chosen to report this case because more than one session was possible and because it offers a better and more thorough explanation of the peculiarity of this type of clinical intervention with EMDR. Julia stayed in hospital longer because of her physical conditions. She had feelings which have led her to the edge of fainting. It was necessary for her to recover a state of sufficient mental and physical wellness in order for the doctors to discharge her. However, this is not the norm because during their clinical activity, the authors often have the chance to perform only one long session with the patient (because, after delivery, mothers stay in hospital only for few days).

During the initial part of the session, the therapist, with Julia's help, collected brief information about 
her personal history and trauma history. In particular, it has to be pointed out that a few years before, Julia had lost her mother because of a tumor and that, since she was a child, her father had always been an alcoholic. Both in the past and at that time, he had never acted like a father to her, he had not been there for her; he had never been an emotional resource for her to count on.

Julia, a Brazilian architect, had come to Italy 5 years before looking for a job. She had suffered from a strong depression after her mother's cancer and subsequent death in less than 2 years (between her last year in Brazil and her first year in Italy). She had been prescribed antidepressants in her country, but at the time of the meeting, she was not taking any medication. She had never undergone psychotherapy treatment. She had been referred to the authors of this article by the hospital staff because of her difficulty in breastfeeding her baby. The words of the midwife who had alerted one of the psychologists of the ward, words that have already been mentioned in the introduction to this article had been "The 12A has been crying since yesterday, she can't hold her baby, she can't breastfeed her . . . she feels faint as soon as the baby latches on . . . can you go and see her?"

The first session, which Julia gladly accepted, took place in her hospital room; her sister was near her sitting on the bed, while the woman sat cross-legged on the bed holding her sleeping baby Sarah. During the course of the session, the woman often looked at her baby although, at times, it seemed like she was about to let go of Sarah.

Julia appeared very tired, with red, puffy eyes because of her crying. She was not crying although, as she told the therapist the story of her pregnancy, and the tone of her voice was thin but clear.

My Italian partner and I have always been very good together. He was already separated when we first met thanks to my work; we have liked each other since the beginning, so we started going out a bit for fun. . . . It took me by surprise. Our relationship kept going and together it got better and better. One day Sergio told me, "I would like to have a baby with you!" I could not believe it, a baby with me! At that point I understood that we were a real couple... I was almost drunk with happiness. And so we started trying.

As the patient told the story of the birth of her baby, the therapist proposed the use of the Narrative with the BLS (the first step of the Breastfeeding and Bonding EMDR Protocol). Julia said,

I got pregnant immediately. I wasn't expecting it to happen so soon. . . . We left anyway for a trip to Bali which we had already planned. I was very careful with everything, but 3 weeks after coming back I had a miscarriage. . . It was awful and I was very upset and saddened. . . . But Sergio has been amazing because he never lost hope. Soon I got pregnant again . . . I was happy . . . a bit anxious initially, but later I felt great, both physically and psychologically. . . . And then it came the time to give birth ... it was painful and trying, but his presence and his caring helped me a lot ... and then Sarah in my arms ... a kind of happiness which I didn't expect and that I had never felt before . . . and then everything collapses because of my breast [crying persistently].

"The pain is unbearable. It is overwhelming." Physically, the woman's breasts were affected by rhagades (small fissures and/ or cracks). "I want to breastfeed, I have always thought I would and never imagined it would be a problem . . . I don't understand what is happening ..." (Her words showed the powerful clash between her expectations and the reality of breastfeeding which was discussed in the previous pages.) Julia said she felt reassured by the presence of her sister who had come to Italy for a month to help and support her.

She is so happy for me ... poor thing ... she can't get pregnant . . . she will start IVF [in vitro fertilization] when she returns to Brazil. As for my father, he is somewhere in Europe, but he is not reliable and he has a drink problem, which got worse when my mother died [crying profusely].

“The pain was too intense ... I can't do it . . ." Julia struggled to tell about the depression which had affected her during her mother's illness and at the time of her death.

I wasn't talking anymore . . . I felt as if I did not exist . . . I was taking medications. . . . Now I am scared of suffering again, but I have to do it . . . I don't understand; everything was going so well ...

Julia calmed down at the end of this anguished and breathless Narrative: "I have to try again to deal with the pain, I have to do it!"

Although the therapist was in the room, the woman was offered a meeting with the midwife to improve breastfeeding. Julia accepted also because 
her husband, who was at that point in the room with her, insisted on it. However, Julia then postponed the meeting to a later date. The therapist ended the session and agreed with her to drop by the day after.

The following day, before the session, the midwives had warned the therapist that Julia the night before had had a severe panic attack and that just the idea of breastfeeding her baby nearly had made her faint.

At the start of the second session, Julia was sitting at a small table for lunchtime, while the baby was sleeping in the cot. Julia was crying desperately and uninterruptedly. Her crying was like a silent scream, her mouth wide open but no sound coming from it. The woman was distressed for not being able to breastfeed.

Julia told the therapist about the "near fainting" of the night before when she had been struggling to breathe. Physically, she described feeling a lump in her throat at the idea of her baby latching onto her breast. She looked very disoriented: On one side, she showed the will to breastfeed her baby, but on the other, she could not and she felt faint. Julia said, "The feeling of a lump in my throat is a complaint which I experienced many times in my life, when I am worried and in distress I have this feeling which leaves me breathless and unable to talk."

The therapist carried on with the EMDR through the identification (with the Google Search) and processing of the moment of greatest difficulty/distress concerning breastfeeding, taking care, and bonding.

Worst image: "I remove the nipple shield ... my breast was covered in blood and there was flesh stuck to the nipple shield ... a piercing pain that cuts through my body" (meanwhile, Julia kept crying in that very peculiar way, like a constant scream, desperate and silent).

NC: Initially, Julia said, "I am deluded." The transition from being deluded to the identification of the NC "I am helpless, I can't breastfeed" required a few steps because feeling deluded for Julia was a highly recurring state of mind in her life. She perceived her inability as if it were a physical limit, some sort of spell, independent of her. At that point, the NC had been established through the request of a PC. This transition produced a shift of focus, from external to internal. Julia reconnected with herself and regained contact with the idea that reality could somehow depend on her, "I can do things . . . I am able to, even if it's hard ... it's painful, but I can do it" (PC). Her mother's illness had brought her to experience an unbearable feeling of incapability and helplessness which had lasted through the whole depressive period and which had become for her a confirmation of her incompetence. This had partly generated that obstructive knot which had made the start of breastfeeding so critical.

NC: "I am helpless (I can't breastfeed)"; PC: "I can do something"; VoC: 1; Emotions: desperation and distress; SUD: 9/10; Location of body sensations: lump in the throat, breathing difficulty

BLS began (eye movements or tapping when Julia was crying). This is what Julia referred (each sentence with inverted commas came after one set of BLS).

My nipples are terrible, my breast is like a cauliflower [Julia gestured with her hands something that was opening but looked frayed].

I am deluded, I thought I had the ability to breastfeed and instead I can't do anything, like when my mother died of cancer ... nothing ...

My breast looks ... yes it really looks ... terrible ... when I saw my mother with the tumor coming out of her, toothless and suffering ... how much suffering. And the tumor coming out of her skin, like an ulcer on her face ... the same ... similar . . to my nipple . . it looked like a frayed and opened cauliflower [Julia gestured again the shape with her hands, while she was crying with her nose running and the tears which filled her eyes and ran down her face profusely].

I understood how much she was suffering ... an immeasurable pain...

The therapist introduced a cognitive intervention to help Julia differentiate between the past (her mother's tumor) and the present (her breast): "What is the difference, for you, between your breast and your mother's tumor?"

Well, regarding the tumor there was nothing left to do, maybe for the breast there is, the breast actually produces milk [she was crying intensely without uttering a word; slowly, her crying weakened until it stopped. Julia was exhausted and sighed deeply many times].

The breast produces milk ... even if ugly and ruined almost like an ulcer, it produces milk ... it is life.

I am not disappointed ... I just need to learn ... . I can do things.

I don't have that feeling of suffocation now. I only feel pain in the uterus ... but I know that it will pass and it's normal [pain in the vulva, uterine cramps].

SUD: $5 / 6$ 
In the meantime, Julia needed to have her gynecological checkup (the doctors' rounds were almost over and the checkup could not be postponed anymore). The second session ended with a sort of positive thinking: The idea of being able to do things, to learn new things (breastfeeding) was emerging. The following day, the therapist saw Julia for the third and last session; she looked more relaxed and confident. The reevaluation of the SUD was 2. The therapist carried on with the sets.

I like to breastfeed, I was able to latch the baby on. It is a bit painful but I breathe and I can control the pain. My breast looks better, it's a breast! When I remove the nipple shield, it doesn't explode anymore. And the baby is very good and she latches on well.

My breast is nicer, even with the nipple shield on: When I take it off it doesn't explode anymore.

It is nice . . . it is not beautiful . . but it is a breast and produces milk, it's not a tumor.

Now I have a feeling of well-being. And I think that, once home, I will breastfeed better and I will feel better [Julia's wish to go home had grown gradually: Initially, she had been afraid of being discharged and sent home "in this state," she had said].

SUD: 1 (ecological); PC: "I can do things (my breast brings life. It is not easy, but I breathe and I can control the pain)"; VoC: 7

Body Scan: "I don't feel any tension in my body now. The pain caused by delivery is less strong now. I'm feeling better [she gave a liberating sigh]. I discovered this way of controlling the pain I feel in the uterus and the breast. I just breathe . . . and even the anxiety, that sometimes still bothers me, disappears."

Once unraveled the main knot, Julia was ready for the last step. The therapist introduced the Installation of Resources, asking her, "What quality or resource would you need the next time you will breastfeed?"

I would need patience, but I am not patient. My mother was patient though . . so patient that she would stand the suffering and the loss of hair [she smiled], so patient that she would let herself be photographed even though she was bold and toothless. . . . And she never stopped smiling. I remember her happy then, despite everything ... I took that picture ... and she managed to be calm, a soothing calm despite everything she was going through ...
These are some of Julia's words between brief sets of BLS.

$\ldots$ and I would probably need new nipples [she laughs]: I need to be patient and learn to accept my breast with the nipple shield ... I think it could help me feel less pain [she was more relaxed now, she did not cry and there was a trace of a smile].

If my mother were here, she would say, "When you are calm you can do everything... this is a beautiful time of your life. ..." And with us [her sister and herself], she was patient and very capable.

After identifying her mother as a model/figure from which to draw the patience and calm needed at this stage, Julia managed to say, "I feel that thinking about my mother's patience and calmness helps me. Thinking of her after these last stages of the treatment feels a bit like having her here with me ..."

The patient returned for follow-up 3 weeks later, and another session with the therapist took place. Julia said, "I like breastfeeding, it helps me untie my knots and the baby is growing."

She was so deeply convinced about this that the woman had carried on nursing her baby, making full use of the skills she had developed and being determined to do it. It is very significant that during her stay in hospital (on the day of the first psychological session) at first she had refused the midwife's offer to deal with her breastfeeding problem but, after the second psychological session, she had decided herself to take it. The drop in the anxiety levels and a higher psychological relaxation and change of perspective had allowed the new mother to pinpoint suitable help and to benefit from it. Julia's choice to breastfeed exclusively was still maintained after 3 weeks (and even 6 weeks after birth, when the therapist had phoned her), despite many attempts from her husband, mother-in-law, and gynecologist to convince her to interrupt breastfeeding. The three of them had insisted on her giving up breastfeeding because, according to them, it was too demanding for her; they had thought she was too tired and psychologically fragile and had not considered breastfeeding as absolutely necessary but replaceable with formula feeding. Therefore, Julia had persisted in breastfeeding despite her medical history that portrayed a woman who, in the past, had been unlikely to oppose anyone else's will, preferring to agree even though it had meant renouncing to take a definite position. Later on, during telephone 
follow-up 6 weeks after delivery, the evaluation of the breastfeeding experience observed a continuous pleasure in the act, even though Julia still felt pain in the breast both during and after a feeding. She reported no physical exertion and high satisfaction of being able to latch the baby, despite the pain and high self-effectiveness. The woman reported a feeling of capability and competence while breastfeeding. Forecasts had shifted from the idea that in the immediate postpartum, breastfeeding would not have last at all to a hypothetical duration "up to 6 months minimum." In the event of a second child, Julia reported a maximum probability of breastfeeding (100\%) also because she hoped "this experience can help me."

\section{Final Comments on the Case}

Julia's perspective had changed. The NC had left space for the recovery of a feeling of self-effectiveness and for the development of her personal resources. The processing of the NC had occurred mainly thanks to the crucial distinction Julia had made: "My breast produces milk . . . even though ugly and ruined almost like an ulcer, it produces milk . . . it brings life."

This is a very important passage. The image of her breast with rhagades and the tactile feeling of it fraying had brought Julia back to her past (the ulcerated tumor on her mother's face), a memory that echoed and transformed itself into an obstructive critical knot. We know how deep rooted the trauma is, how imprinted in a person's neural network, and how it shows itself in its sensory form. And in Julia's case, we have the confirmation of this.

During follow-up, Julia talked about her well-being linked to

experiencing the satisfaction of being able to [breastfeed], even though it is not easy. I can do things, despite the obstacles encountered; I find it difficult, it is not immediate, but I can stand the struggle thanks to the patience which I find inside me, the calmness and the importance of what I do . . . and plus it is so wonderful!

At that stage, the calmness and the patience, represented by the maternal model were not just something she wished for but something she could personally experience. The actual effectiveness of her actions was also confirmed by the fact that the gain in weight of her baby was absolutely adequate. The competence, she felt, enabled her to stay firmly convinced of her choice of carrying on with breastfeeding, trusting herself and not letting other people (although insistent) influence her. She was strongly advised to undergo therapy to deal with the events that had had a negative influence on her during that phase of her life (difficulties with her father, the pain of her mother's death, etc.).

\section{Discussion}

As it was already shown, the Protocol allows working on the patient's blocks effectively because it identifies the traumatic memories triggered by the situation of breastfeeding and points out the mother's critical knots. It would be useful to have some clarification on the Breastfeeding and Bonding EMDR Protocol. The Protocol originates from the use of EMDR on recent events, in particular, it relates to the R-TEP (E. Shapiro, 2009a; E. Shapiro \& Laub, 2009) but differs from it. The scope of use is quite different: The Breastfeeding and Bonding EMDR Protocol does not start from a traumatic event, which suddenly overwhelms a person's life. Instead, we are talking about a positive situation, that of the birth of a healthy child that the woman, who is met by the clinical psychologist, wants to breastfeed. Unless psychological problems related to breastfeeding are thoroughly known by the psychologist, he might think that it is just a peak performance, which can be dealt with mainly using RDI. Instead, EEI with the Breastfeeding and Bonding EMDR Protocol allows the therapist to face the woman's crisis more effectively, first by decreasing the level of arousal and then working on what we have defined as critical knots in a targeted manner, taking into account the NCs, emotions, and physical sensations that link the situation of breastfeeding to the previous life experiences of the woman. Regarding the clinical case, if Julia had not been able to connect her breastfeeding problems with her mother illness in a very specific, targeted way, identifying consequently also the differences between past and present time, the emotional obstacles would have been presumably too serious for her to continue breastfeeding.

NCs can be very specific to the situation of breastfeeding, and this is another peculiarity of the Protocol. NCs allow to identify critical knots in a focused manner; critical points often reflect the mother's feeling of being one with her child, and what happens to the baby, it is as if it happened directly to her. If her child is perceived to be in danger during the processing, she will feel in danger too, if her child is seen as defective, 
she will think to be defective too because she gave birth to him or her. In several cases, at the beginning of the processing, it is most effective to start with an $\mathrm{NC}$ on the child and during the processing get to the negative belief about self (and almost always, this is really what happens in the clinical experience of the authors of the article).

When using telescopic processing (E. Shapiro \& Laub, 2009) from EMD to EMDr and EMDR, EMD can be often less relevant and effective with respect to several moments of greatest difficulty/distress and can require more often the implementation of EMDr and then EMDR after a few sets of BLS, broadening the focus of approach, because there is not any major trauma (any "big T trauma") to desensitize as first step of the intervention. If it is useful to let the connections with other experiences emerge to clarify the origins of the critical knots, the therapist should pay attention on when to return to the target to not lose tight focus on the issue.

There are cases in which it is inappropriate to use the Protocol (or it is recommended at least to postpone its use in a later stage, if it is possible to have more sessions). First of all, when the baby is born with medical problems (but the mother can still breastfeed him or her): a syndrome, an illness of some kind, a significant prematurity. In these cases, the focus will be on the trauma related to the child's disease or premature birth, even if the mother has started breastfeeding her newborn and some difficulties have arisen. If the birth has been traumatic, the focus of intervention will be on the birth trauma as a recent traumatic event (Cattaneo, Roveraro, Chiorino, Salerno, et al., 2015). If during pregnancy the woman had a major trauma, "a big T trauma," and it is evident that she has developed symptoms of posttraumatic stress disorder related to that event (or acute stress disorder if less than 1 month passed), the priority is given to that trauma.

The case presented here is specific to a situation where the critical knot is generated by the connection between the breastfeeding experience and a past "big T trauma." In other cases, the critical knot may be less evident and be identified more slowly while applying the protocol, for example, when the critical experiences that breastfeeding triggers are light attachment traumas, which are particularly relevant when you become a caregiver. The critical knot may also originate during conception and/or pregnancy: an uncertain diagnosis of fetal disease, later disconfirmed by prenatal exams, as well as a long history of failures with MAP or heterologous fertilization, or again previous miscarriages in the first trimester may have left deep marks on the woman.

Should a woman maintain high SUD level, should she remain very anxious or in a state of strong crisis, the clinical psychologist will discuss the case with the hospital staff: Could the discharge be postponed so that the psychologist can have one more session with the woman the day after and reconsider her conditions? Is a psychiatrist's intervention needed? Is it possible to fix an appointment with the psychologist in the postpartum office in the hospital within a couple of days after discharge?

In the authors' clinical experience, the Protocol can be usefully applied even when the woman is already back home with her baby; for example, a psychologist can meet her for the first time few days after discharge in the postpartum medical office or in the breastfeeding medical office in the hospital, in a family consultation center, or in private practice.

Obviously, this is a single-case study, and this is the limitation of this study itself. The limitations of the Protocol, instead, are related to the fact that if there are any experiences, which were dysfunctionally stored in the memory of the woman and which have hindered breastfeeding, the intervention, short, targeted as it is, can stop the hindrance causing positive consequences during this sensitive time of her life, but the effects of these traumatic memories could recur in later phases.

Future research should include a randomized controlled trial to study the effects of implementing the Protocol and compare it with other psychological interventions related to breastfeeding, which are principally based on generic supportive intervention and self-efficacy promotion (Burns et al., 2012; Dyson et al., 2006; Kingston et al., 2007; Renfrew et al., 2012; Schmied et al., 2011). There should be follow-up to observe breastfeeding and if and how it changes (exclusive breastfeeding, predominant breastfeeding, mixed feeding, or artificial feeding) and bonding (e.g., using the Mother-Infant Bonding Scale before the psychological intervention and during follow-up) according to the type of intervention implemented.

\section{Conclusion}

The Breastfeeding and Bonding EMDR Protocol has been devised and developed precisely as a prompt, focused, and "nonpathologizing" intervention. If the psychologist has the "tools," and the Protocol can be a very significant one, to be effective and to find the right channel quickly, in a situation with so much changing potential, the woman can untie a few knots 
and "the crisis event" becomes a transformative, fruitful time with powerful effects on breastfeeding and bonding and with highly preventive value both for the woman and the newborn and, in broader terms, for the couple and the family system.

\section{References}

Adewale, O. R. (2006). The lived experience of first-time breastfeeding mothers. International Journal of Childbirth Education, 21(3), 21-25.

American Academy of Pediatrics. (2014). Breastfeeding handbook for physicians (2nd ed.). Elk Grove Village, IL: Author.

Ballard, C. G., Stanley, A. K., \& Brockington, I. F. (1995). Post-traumatic stress disorder (PTSD) after childbirth. The British Journal of Psychiatry, 166(4), 525-528.

Barber, C. M., Abernathy, T., Steinmetz, B., \& Charlebois, J. (1997). Using a breastfeeding prevalence survey to identify a population for targeted programs. Canadian Journal of Public Health, 88(4), 242-245.

Beck, C. T. (2006). Postpartum depression: It isn't just the blues. The American Journal of Nursing, 106(5), 40-50.

Bernstein, E. M., \& Putnam, F. W. (1986). Development, reliability, and validity of a dissociation scale. The Journal of Nervous and Mental Disease, 174(12), 727-735.

Brodribb, W. (2012). Breastfeeding management in Australia. Melbourne, Victoria, Australia: Australian Breastfeeding Association.

Burns, E., Schmied, V., Fenwick, J., \& Sheehan, A. (2012). Liquid gold from the milk bar: Constructions of breastmilk and breastfeeding women in the language and practices of midwives. Social Science \& Medicine, 75(10), 1737-1745.

Buydens, S., Wilensky, M., \& Hensley, B. J. (2014). Effects of the EMDR protocol for recent traumatic events on acute stress disorder: A case series. Journal of EMDR Practice and Research, 8(1), 2-12.

Cattaneo, M. C., Roveraro, S., Chiorino, V., Macchi, E. A., Salerno, R., Gatti, M., . . Mosca, F. (2015). The behavior over time of postnatal depression symptomatology and its early detection via the Edinburgh Postnatal Depression Scale: An Italian longitudinal study. International Journal of Advanced Nursing Studies, 4(1), 23-29.

Cattaneo, M. C., Roveraro, S., Chiorino, V., Salerno, R., Macchi, E. A., \& Bertolucci, G. G. (2015, July). Negative cognitions for perinatal psychology in "When birth becomes crisis: Taking care of the primary attachment relationship in a maternity ward." Paper presented at the EMDR Europe Conference, Milan, Italy.

Coates, R., Ayers, S., \& de Visser, R. (2014). Women's experiences of postnatal distress: A qualitative study. BMC Pregnancy and Childbirth, 14(1), 359.

Colin, W. B., \& Scott, J. A. (2002). Breastfeeding: Reasons for starting, reasons for stopping and problems along the way. Breastfeeding Review, 10(2), 13-19.
Cousineau, T. M., Green, T. C., Corsini, E. A., Barnard, T., Seibring, A. R., \& Domar, A. D. (2006). Development and validation of the Infertility Self-Efficacy scale. Fertility and Sterility, 85(6), 1684-1696.

Dyson, L., McCormick, F., \& Renfrew, M. J. (2006). Interventions for promoting the initiation of breastfeeding. Evidence-Based Child Health: A Cochrane Review Journal, 1(2), 592-616.

Else-Quest, N. M., Hyde, J. S., \& Clark, R. (2003). Breastfeeding, bonding, and the mother-infant relationship. Merrill-Palmer Quarterly, 49, 495-517.

Fernandez, I., Callerame, C., Maslovaric, G., \& Wheeler, K. (2014). EMDR Europe humanitarian programs: Development, current status, and future challenges. Journal of EMDR Practice and Research, 8(4), 215-224.

Francis, D. D., Champagne, F. C., \& Meaney, M. J. (2000). Variations in maternal behavior are associated with differences in oxytocin receptor levels in the rat. Journal of Neuroendocrinology, 12(12), 1145-1148.

Glasheen, C., Richardson, G. A., \& Fabio, A. (2010). A systematic review of the effects of postnatal maternal anxiety on children. Archives of Women's Mental Health, 13(1), 61-74.

Groer, M. W., Davis, M. W., \& Hemphill, J. (2002). Postpartum stress: Current concepts and the possible protective role of breastfeeding. Journal of Obstetric, Gynecologic, \& Neonatal Nursing, 31(4), 411-417.

Guedalia, G. S. B., \& Yoeli, F. R. (2009). EMDR emergency room and wards protocol (EMDR-ER). In M. Luber (Ed.), Eye movement desensitization and reprocessing (EMDR) scripted protocols: Basics and special situations (pp. 241-250). New York, NY: Springer Publishing.

Hailes, J. F., \& Wellard, S. J. (2000). Support for breastfeeding in the first postpartum month: Perceptions of breastfeeding women. Breastfeeding Review, 8(3), 5-9.

Hauck, Y., Hall, W. A., \& Jones, C. (2007). Prevalence, selfefficacy and perceptions of conflicting advice and selfmanagement: Effects of a breastfeeding journal. Journal of Advanced Nursing, 57(3), 306-317.

Hauck, Y., Langton, D., \& Coyle, K. (2002). The path of determination: Exploring the lived experience of breastfeeding difficulties. Breastfeeding Review, 10(2), 5-12.

Hegney, D., Fallon, T., \& O’Brien, M. L. (2008). Against all odds: A retrospective case-controlled study of women who experienced extraordinary breastfeeding problems. Journal of Clinical Nursing, 17(9), 1182-1192.

Hill, P. D. (1991). The enigma of insufficient milk supply. The American Journal of Maternal Child Nursing, 16(6), 312-316.

Jansen, J., de Weerth, C., \& Riksen-Walraven, J. M. (2008). Breastfeeding and the mother-infant relationship-a review. Developmental Review, 28(4), 503-521.

Jarero, I., Artigas, L., Uribe, S., \& Miranda, A. (2014). EMDR therapy humanitarian trauma recovery interventions in Latin America and the Caribbean. Journal of EMDR Practice and Research, 8(4), 260-269. 
Kim, P., Feldman, R., Mayes, L. C., Eicher, V., Thompson, N., Leckman, J. F., \& Swain, J. E. (2011). Breastfeeding, brain activation to own infant cry, and maternal sensitivity. Journal of Child Psychology and Psychiatry, 52(8), 907-915.

Kingston, D., Dennis, C. L., \& Sword, W. (2007). Exploring breast-feeding self-efficacy. Journal of Perinatal \& Neonatal Nursing, 21, 207-215.

Kuzela, A. L., Stifter, C. A., \& Worobey, J. (1990). Breastfeeding and mother-infant interactions. Journal of Reproductive and Infant Psychology, 8, 185-194.

Lavelli, M., \& Poli, M. (1998). Early mother-infant interaction during breast- and bottlefeeding. Infant Behavior \& Development, 21, 667-683.

Leeds, A. M., \& Shapiro, F. (2000). EMDR and resource installation: Principles and procedures for enhancing current functioning and resolving traumatic experiences. In J. Carlson \& L. Sperry (Eds.), Brief therapy strategies with individuals and couples (pp. 469-534). Phoenix, AZ: Zeig, Tucker \& Theisen.

Li, R., Fein, S. B., Chen, J., \& Grummer-Strawn, L. M. (2008). Why mothers stop breastfeeding: Mothers' selfreported reasons for stopping during the first year. Pediatrics, 122(Suppl. 2), S69-S76.

Luber, M., \& Shapiro, F. (2009). The safe/calm place protocol. In M. Luber (Ed.), Eye movement desensitization and reprocessing (EMDR) scripted protocols: Basics and special situations (pp. 67-70). New York, NY: Springer Publishing.

Madrid, A., Skolek, S., \& Shapiro, F. (2006). Repairing failures in bonding through EMDR. Clinical Case Studies, (5), 271-286.

Mann, P. E., \& Bridges, R. S. (2001). Lactogenic hormone regulation of maternal behavior. Progress in Brain Research, 133, 251-262.

Matthey, S., Barnett, B., Ungerer, J., \& Waters, B. (2000). Paternal and maternal depressed mood during the transition to parenthood. Journal of Affective Disorders, 60(2), 75-85.

Maxfield, L. (2008). EMDR treatment of recent events and community disasters. Journal of EMDR Practice and Research, 2(2), 74-78.

Mezzacappa, E. S., Guethlein, W., Vaz, N., \& Bagiella, E. (2000). A preliminary study of breast-feeding and maternal symptomatology. Annals of Behavioral Medicine, 22(1), 71-79.

Missonnier, S. (2003). La consultation thérapeutique périnatale. Un psychologue à la maternité [Perinatal Therapeutic Treatment]. Toulouse, France: Éditions Érès.

Nicol-Harper, R., Harvey, A. G., \& Stein, A. (2007). Interactions between mothers and infants: Impact of maternal anxiety. Infant Behavior \& Development, 30(1), 161-167.

O’Hara, M. W., \& Swain, A. M. (1996). Rates and risk of postpartum depression-a meta-analysis. International Review of Psychiatry, 8(1), 37-54.
Parnell, L. (2007). A therapist's guide to EMDR: Tools and techniques for successful treatment. New York, NY: Norton.

Renfrew, M. J., McCormick, F. M., Wade, A., Quinn, B., \& Dowswell, T. (2012). Support for healthy breastfeeding mothers with healthy term babies. Cochrane Database of Systematic Reviews, (5), CD001141.

Riem, M. M., Pieper, S., Out, D., Bakermans-Kranenburg, M. J., \& van IJzendoorn, M. H. (2011). Oxytocin receptor gene and depressive symptoms associated with physiological reactivity to infant crying. Social Cognitive and Affective Neuroscience, 6(3), 294-300.

Rodrigues, S. M., Saslow, L. R., Garcia, N., John, O. P., \& Keltner, D. (2009). Oxytocin receptor genetic variation relates to empathy and stress reactivity in humans. Proceedings of the National Academy of Sciences, USA, 106(50), 21437-21441.

Ross, L. E., McLean, L. M. (2006). Anxiety disorders during pregnancy and the postpartum period: A systematic review. Journal of Clinical Psychiatry, 67(8), 1285-1298.

Schmied, V., Beake, S., Sheehan, A., McCourt, C., \& Dykes, F. (2011). Women's perceptions and experiences of breastfeeding support: A metasynthesis. Birth, 38(1), 49-60.

Shapiro, E. (2009a). EMDR treatment of recent trauma. Journal of EMDR Practice and Research, 3(3), 141-151.

Shapiro, E. (2009b). Four elements exercise for stress management. In M. (Ed.), Eye movement desensitization and reprocessing (EMDR) scripted protocols: Basics and special situations (pp. 73-80). New York, NY: Springer Publishing.

Shapiro, E., \& Laub, B. (2009). The recent-traumatic episode protocol (R-TEP): An integrative protocol for early EMDR intervention (EEI). In M. Luber (Ed.), Eye movement desensitization and reprocessing (EMDR) scripted protocols: Basics and special situations (pp. 251-270). New York, NY: Springer Publishing.

Shapiro, F. (2001). Eye movement desensitization and reprocessing (EMDR): Basic principles, protocols, and procedures (2nd ed.). New York, NY: Guilford Press.

Siddiqui, A., Hagglof, B., \& Eisemann, M. (2000). Own memories of upbringing as a determinant of prenatal attachment in expectant women. Journal of Reproductive and Infant Psychology, 18, 67-74.

Solomon, R. M. (2006, September). The art of EMDR: Dealing with abreactions. Paper presented at the 11th EMDR International Association Conference, Philadelphia, PA.

Solomon, R. M., \& Shapiro, F. (2008). EMDR and the adaptive information processing model: Potential mechanisms of change. Journal of EMDR Practice and Research, 2(4), 315-325.

Tharner, A., Luijk, M. P., Raat, H., van IJzendoorn, M. H., Bakermans-Kranenburg, M. J., Moll, H. A., . . Tiemeier, H. (2012). Breastfeeding and its relation to maternal sensitivity and infant attachment. Journal of Developmental \& Behavioral Pediatrics, 33(5), 396-404. 
Wiesenfeld, A. R., Malatesta, C. Z., Whitman, P. B., Granrose, C., \& Uili, R. (1985). Psychophysiological response of breast- and bottle-feeding mothers to their infants' signals. Psychophysiology, 22(1), 79-86.

Williamson, I., Leeming, D., Lyttle, S., \& Johnson, S. (2012). 'It should be the most natural thing in the world': Exploring first-time mothers' breastfeeding difficulties in the UK using audio-diaries and interviews. Maternal of Child Nutrition, 8(4), 434-447.
Winnicott, D. W. (1987). Babies and their mothers. London, United Kingdom: Free Association Books.

World Health Organization. (2016). Infant and young child feeding. Geneva, Switzerland: Author. Retrieved from http:// www.who.int/entity/mediacentre/factsheets/fs342/en/

Correspondence regarding this article should be directed to Valentina Chiorino, Mangiagalli Hospital, Via della Commenda 12, Milan, Italy. E-mail: valentina.chiorino@tin.it 


\section{SPRINGER/PUBLISHING COMPANY}

\section{These new and forthcoming titles are the perfect additions to any EMDR practioner's resources. Visit www.springerpub.com for more information.}

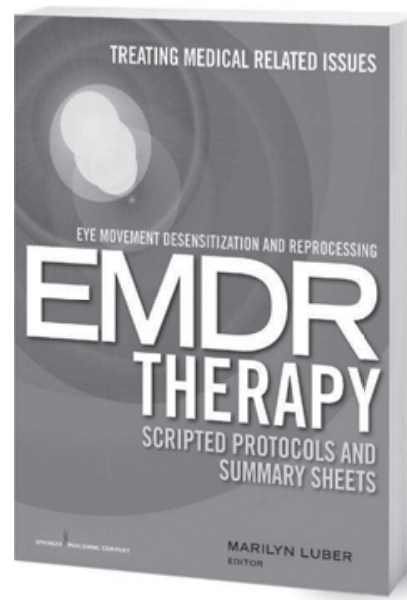

Marilyn Luber PhD

9780826194213

March 2017

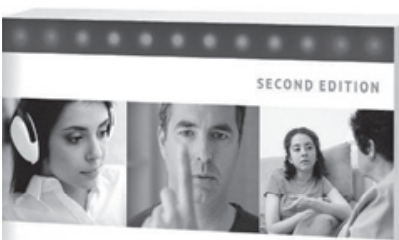

A Guide to the

Standard EMDR

Therapy Protocols for

Clinicians, Supervisors, and Consultants

Andrew M. Leeds

Andrew M. Leeds PhD

9780826131164

February 2016

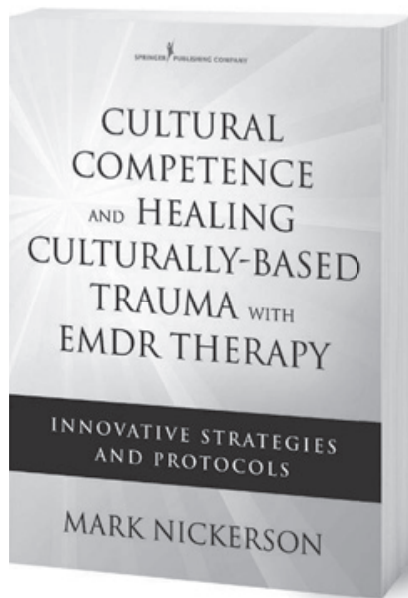

Mark Nickerson LICSW

9780826142863

August 2016

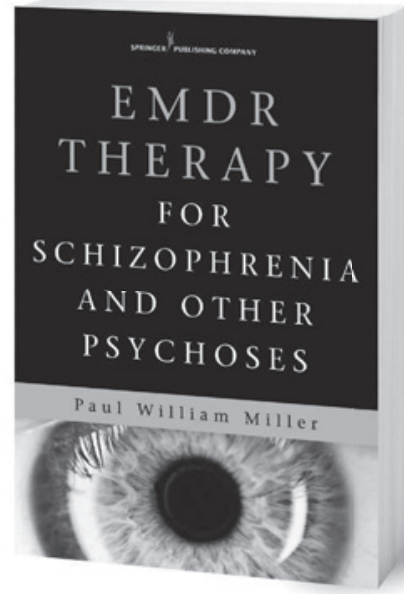

Paul Miller MD, DMH, MRCPsych

9780826123176

October 2015

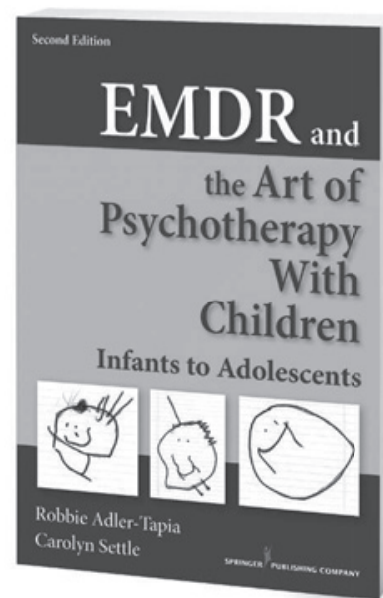

Robbie Adler-Tapia PhD

Carolyn Settle MSW, LCSW

9780826138019

August 2016

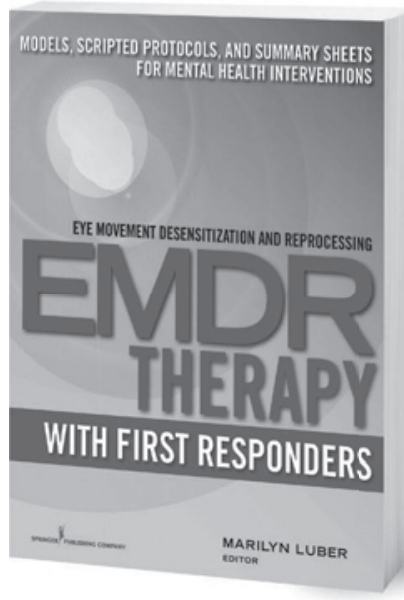

Marilyn Luber PhD

9780826133380

September 2015

11 West 42nd Street, 15th FL, New York, NY 10036 • Fax: 212-941-7842

Order Toll-Free: 877-687-7476 • Order online: www.springerpub.com 